\title{
Heat Equation on Phase Space and the Classical Limit of Quantum Mechanical Expectation Values
}

\author{
A. Grossmann \\ Centre de Physique Théorique, C.N.R.S., F-13274 Marseille Cedex 2, France \\ R. Seiler \\ Freie Universität Berlin, FB 20, Physik, D-1000 Berlin 33
}

\begin{abstract}
The expectation value of a quantum mechanical operator, taken in coherent states and suitably rescaled, is the solution of an initial value problem for the heat equation on phase space, in which $\hbar$ plays the role of time, and the classical observable is the distribution of temperature at $\hbar=0$.
\end{abstract}

\section{Introduction}

A recent paper by Hepp [1] is devoted to the classical limit of (rescaled) expectation values in coherent states and to their time evolution. Here we sharpen some results of [1] by relating the classical limit to an initial value problem in $\hbar$. This is done with the help of a quantization formula derived in [2].

\section{Notations}

Denote by $E$ a $2 v$-dimensional real vector space with a symplectic form $\sigma$. (Phase space for $v<\infty$ degrees of freedom.) Elements of $E$ will be denoted by $a, b, v \ldots$ Fix on $E$ a $\sigma$-allowed complex structure $J$, i.e. a linear map satisfying $J^{2}=-1$, $\sigma(J a, J v)=\sigma(a, v)$ and $\sigma(a, J a)>0$ for $a \neq 0$. Introduce the orthogonal form $s(a, v)=$ $\sigma(a, J v)$, and the (phase space) Gaussian $\Omega(v)=e^{-\pi s(v, v)}$. Normalize the invariant measure $d v$ on $E$ by the requirement $\int \Omega(v) d v=1$. This is equivalent to the requirement $F^{2}=1$ where $F$ is the symplectic Fourier transform:

$$
F f)(v)=\tilde{f}(v)=\int e^{2 i \pi \sigma\left(v, v^{\prime}\right)} f\left(v^{\prime}\right) d v^{\prime} .
$$

In the Hilbert space $L^{2}(E ; d v)$ consider the family of functions $\Omega^{a}$ :

$$
\Omega^{a}(v)=e^{-2 i \pi \sigma(a, v)} \Omega(v+a) .
$$

Denote by $\mathscr{H}$ the closed linear span of the family $\Omega^{a}$, with the scalar product inherited from $L^{2}(E ; d v)$. For any $\Phi \in \mathscr{H}$ one has $\left(\Omega^{a}, \Phi\right)=k \Phi(-a)$, with

$$
k=(\Omega, \Omega)=2^{-v} .
$$


Also, for every $\Phi \in \mathscr{H}$ one has $F \Phi=M \Phi$, where $M$ is the parity operator:

$$
(M \Phi)(v)=\Phi(-v) \text {. }
$$

Define $(W(a) \Phi)(v)=e^{-2 i \pi \sigma(a, v)} \Phi(v+a)$. The Weyl operators $W(a)$ act irreducibly in $\mathscr{H}$.

A convenient way of writing (even very unbounded) linear operators $A$ in $\mathscr{H}$ is to consider the associated kernel: $A(a, b)=\left(\Omega^{a}, A \Omega^{b}\right)$. One proves then

$(A \Phi)(a)=\left(1 / k^{2}\right) \int A(-a,-b) \Phi(b) d b$.

\section{Weyl Quantization}

It consists in associating, to a function $f_{c}$ on phase space, the operator $Q\left(f_{c}\right)$ defined formally by

$$
Q\left(f_{c}\right)=\int \tilde{f}_{c}(v) W(-v / 2) d v=\int f_{c}(v / 2) W(v) M d v .
$$

It has been shown in [2] that the two expressions coincide. In order to avoid a discussion of the convergence of the operator-valued integrals, we replace (1) by the kernel

$$
\left(\Omega^{a}, Q\left(f_{c}\right) \Omega^{b}\right)=\int \tilde{f_{c}}(v) \Omega^{(a, b)}(-v / 2) d v=\int f_{c}(v / 2) \Omega^{(a,-b)}(v) d v
$$

where

$$
\Omega^{(a, b)}(v)=\left(\Omega^{a}, W(v) \Omega^{b}\right)=k e^{2 i \pi \sigma(b, a)} e^{-2 i \pi \sigma(a+b, v)} \Omega(v-a+b) .
$$

\section{Heat Equation on Phase Space}

Define the Laplacian, $\Delta$, on $E$, by $\Delta=-F_{S} F$, where $s$ is the operator of multiplication by $s(v, v)$. Consider on $E$ the heat equation:

$$
\partial f / \partial \hbar=(\pi / 4) \Delta f \text {. }
$$

Let $f_{c}$ be a function in the uniqueness and correctness class for (4); this is only a very mild requirement. Define $f(\hbar, v)$ as the solution of $(4)$, with initial data $f_{c}(v)$. In other words, $f(\hbar, v)$ is the distribution of "temperature" at "time" $\hbar$, resulting from an initial distribution $f(0, v)=f_{c}(v)$.

Theorem. One has

$$
f(\hbar, v)=(1 / k)\left(\Omega^{\hbar-\frac{1}{2} v}, \quad Q\left(f_{c}\left(\hbar^{\frac{1}{2}} \cdot\right)\right) \Omega^{\hbar-\frac{1}{2} v}\right)
$$

where $f_{c}\left(\hbar^{\frac{1}{2}} \cdot\right)$ is the function $v \rightarrow f_{c}\left(\hbar^{\frac{1}{2}} v\right)$.

Equation (5) describes very intuitively the way in which a (suitably rescaled) matrix element tends to a classical function. We shall apply it in a forthcoming paper to the study of time evolution.

In order to prove (5), specialize (2) to

$\left(\Omega^{a}, Q(f) \Omega^{a}\right)=k \int f(v / 2) \Omega(v-2 a) d v$ 
and notice that $G_{\lambda}(v)=\lambda^{-v} \Omega\left(\lambda^{-\frac{1}{2}} v\right)$ is the elementary solution of the heat equation $\partial G / \partial \lambda=\pi \Delta G$.

It is possible to derive analogous equations for off-diagonal matrix elements, and equations in which the initial data are given by $\tilde{f}_{c}$.

Acknowledgement. This work was partially supported by contract SE 2871 of the Deutsche Forschungsgemeinschaft.

\section{References}

1. Hepp, K.: Commun. math. Phys. 35, 265 (1974)

2. Grossmann, A.: Commun. math. Phys. 48, 191-194 (1976)

Communicated by H. Araki

Received November 10, 1975 
